\title{
Ductal carcinoma in-situ in Turner syndrome patient undergoing hormone replacement therapy: A case report
}

\author{
Rashmi Bawa1, Praise Matemavi², Ilan Maizlin², Kap-Jae Sung2 \\ ${ }^{1}$ Department of Surgery, Pine Health Services, Maine, USA \\ 2Department of Surgery, New York Presbyterian Queens Hospital/Weill Cornell Medical College, New York, USA
}

Received October 01, 2015; Revised January 17, 2016; Accepted January 18, 2016; Published Online February 06, 2016

\section{Case Report}

\begin{abstract}
Turner's syndrome is a rare congenital disease which affects about 1 in every 2500-3000 live-born females. This happens due to chromosomal abnormalities in a phenotypic female, causing increased gonadotropin concentrations and low concentrations of estrogens from infancy. As a result, hormone replacement therapy is started in most adolescent Turner syndrome patients to initiate and sustain sexual maturation. Accordingly, most Turner's syndrome patients undergo several decades of estrogen replacement therapy, from puberty to post-menopausal age. The highly publicized findings of the Women's Health Initiative have called into question the appropriateness of hormone replacement therapy in adolescents with Turner's syndrome. Those concerns were mostly theoretical extrapolations, as few prospective studies of cancer occurrence in women with Turner syndrome have been reported. Consequently, several recent publications have challenged those extrapolations, based on the assertion that the levels of hormone replacement in Turner syndrome patients are well below the physiologic levels observed in normal menstruating women, as well as the fact that these women are significantly younger than those studied by the Women's Health Initiative. In discord to those reports, we present a case of ductal carcinoma in-situ in a 40-year-old Turner patient, who had undergone over two decades of combined hormone replacement therapy. The patient underwent an elective excisional biopsy for a palpable mass, with histopathology revealing a complex fibroadenoma with a nidus of ductal carcinoma in-situ. The lesion was noted to be estrogen receptor positive and progesterone receptor negative, with heavy staining for HER-2/Neu receptor. The patient was treated with tamoxifen. While a rare case, it is imperative for the astute clinician to keep in mind the consequences of long-term hormone replacement therapy in Turner's syndrome patients in order to avoid missed diagnosis of breast cancer for optimum management of these patients.
\end{abstract}

Keywords: Turner Syndrome; Ductal Carcinoma In-Situ; DCIS; HRT; Estrogen Replacement Therapy; Breast Cancer

\section{Introduction}

Turner's syndrome (TS), a rare congenital disease affecting about 1 in every $2500-3000$ live-born females. ${ }^{1}$ TS is the result of chromosomal abnormalities in a phenotypic female. These patients have characteristic clinical features and specific somatic abnormalities, the most consistent being short stature, ovarian dysgenesis with concomitant primary amenorrhea ${ }^{2}$. Complete $45, \mathrm{X}$ monosomy accounts for $40-60 \%$ of the karyotypes, whereas $5-10 \%$ of patients have a duplication of the long arm of one $\mathrm{X}$ (isochromosome $\mathrm{Xq}$ ) and most of the remaining karyotypes show a mosaicism. ${ }^{2}$ The consequent phenotype is thought to be the result of haploinsufficiency of genes on the $\mathrm{X}$ chromosome that escape X-inactivation in early embryogenesis. ${ }^{3}$ Women with Turner syndrome have increased gonadotropin concentrations from infancy and low concentrations of estrogens. As a result, hormone replacement therapy (HRT) is started in most adolescent Turner syndrome patients to initiate and sustain sexual maturation. ${ }^{4}$ HRT is usually maintained throughout the patient's adult life. It is crucial to the maintenance of tissue and bone integrity and has positive effects on the development of Corresponding author: Rashmi Bawa; Department of Surgery, Pine Health Services, Maine, USA.

Cite this article as: Bawa R, Matemavi P, Maizlin I, Sung KJ. Ductal carcinoma in-situ in Turner syndrome patient undergoing hormone replacement therapy: A case report. Int J Cancer Ther Oncol. 2016; 4(1):4113. DOI: 10.14319/ijcto.41.13 
patients with TS. ${ }^{5}$ Accordingly, most TS patients undergo several decades of estrogen replacement therapy, from pubertal to post-menopausal age.

The highly publicized findings of the Women's Health Initiative (WHI) have revealed the risks of combined progestin-estrogen therapy in postmenopausal women, including increased risk of certain neoplasms, such as endometrial and breast malignancies. ${ }^{4}$ More specifically, It has been reported that there is an increased risk of breast cancer in healthy postmenopausal women who receive more than 5 years of HRT. ${ }^{6}$ The extrapolation of these findings to younger women has called into question the appropriateness of HRT in adolescents with TS. Those concerns and generalizations were theoretical as few prospective studies of cancer occurrence in women with Turner syndrome have been reported. ${ }^{7}$

Moreover, several recent publications ${ }^{4,8}$ have challenged these claims that long-term hormone-replacement therapy does not increase the risk of breast cancer in women with TS. The authors of those publications base their assertions on the fact that the levels of hormone replacement in TS are well below the physiologic levels observed in normal menstruating women and in women receiving oral contraceptives. ${ }^{4}$ Similarly, they claim that the mean age of WHI subjects was 63 years, while it is unclear whether these findings are applicable to a younger population. ${ }^{8}$

In discord to those reports, we present a case of ductal carcinoma in-situ (DCIS) in a 40-year-old Turner patient, who had undergone over two decades of combined hormone replacement therapy and was found to have left breast ductal carcinoma in-situ. While a rare case, it is imperative for the astute clinician to keep in mind the consequences of long-term hormone replacement therapy in Turner's syndrome patients to avoid delay in diagnosis and treatment of breast cancer and for the optimum management of these patients.

\section{Case presentation}

40-year-old female diagnosed with Turner syndrome at the age of 13, started on hormonal therapy with estrogen and progesterone at the age of 16 years for growth and development with appropriate pubertal development by 17 years of age. She was diagnosed with streak ovaries and underwent bilateral oophorectomy for increased risk of ovarian cancer. The patient continued on long term hormonal replacement therapy for 23 years.

The patient presented to our Breast Center for evaluation after she noted a palpable lump in the upper outer quadrant of the left breast. She denied any prior history of palpable breast masses or abnormal findings on routine mammographic or ultrasound screening studies. There was no associated breast pain or tenderness, as well as any recent or remote history of trauma to the breast. Mammographic and ultrasound investigations during initial work-up demonstrated a smooth solid mass in the left upper, outer quadrant, approximately $2.5 \mathrm{~cm}$ in diameter (Figure 1).
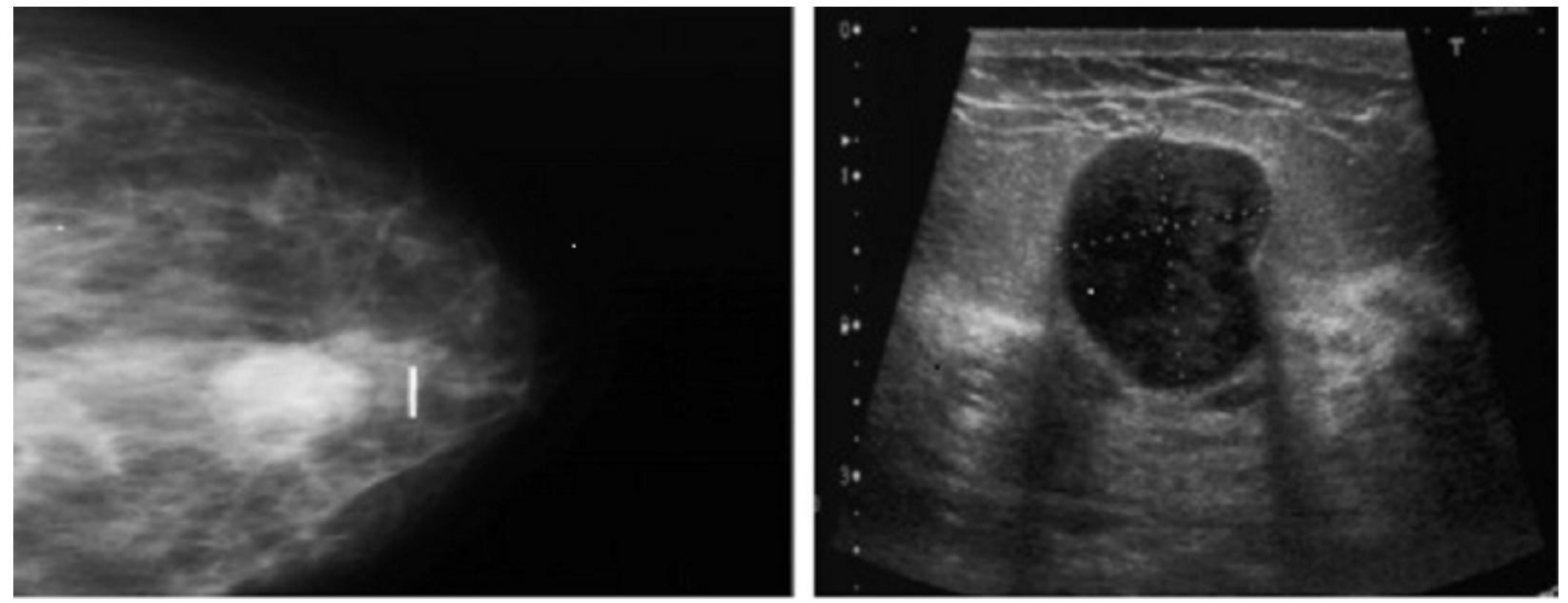

Figure 1: Left Mammogram left breast smooth solid mass, right left breast ultrasound $2.5 \mathrm{~cm}$ heterogeneous mass. 


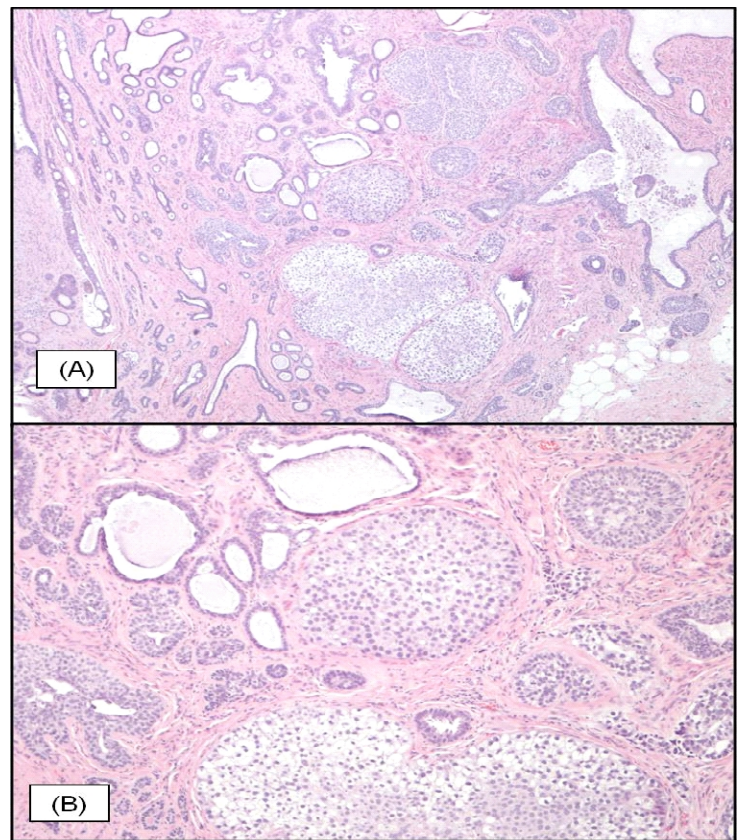

Figure 2: H\&E histology of the breast lesion demonstrating (a) fibroadenoma with a nidus of ductal carcinoma in-situ of solid type (10x magnification), with (b) apocrine and clear cell features and intermediate nuclear grade $(40 \mathrm{x}$ magnification).

The patient underwent an elective excisional biopsy, during which the lesion was removed en-masse with appropriate gross margins. She tolerated the procedure well and her recovery was uneventful.

Pathology came back as fibroadenoma with DCIS (Figure 2). After counselling the patient about treatment options a decision was reached to start her on tamoxifen. Radiation therapy was considered and discussed at the facility's multi-disciplinary oncological conference but there was no evidence to support benefit for DCIS that was confined to the fibroadenoma with an appropriate negative margin. The patient did well with tamoxifen. At two year follow up, the patient has no recurrence of disease clinically and radiologically.

\subsection{Pathological features}

The resection specimen consisted of one irregular piece of fibrous, fatty breast tissue measuring $4.2 \times 3.4 \times 2.0$ $\mathrm{cm}$. Histology confirmed a complex fibroadenoma, with serial sections revealing an extensive pink-white, indurated fibrous area (Figure 2) measuring $3.0 \mathrm{~cm}$ in greatest dimension, with the remainder of the cuts demonstrating yellow, lobular fatty tissue. The fibroadenoma was initially considered to be secondarily involved with atypical ductal hyperplasia. However, when the slides of atypical ductal hyperplasia were stained with both routine H\&E (Hematoxylin and eosin) staining and immunostaining for androgen receptor, review of those slides (Figure 3) confirmed the presence of DCIS.

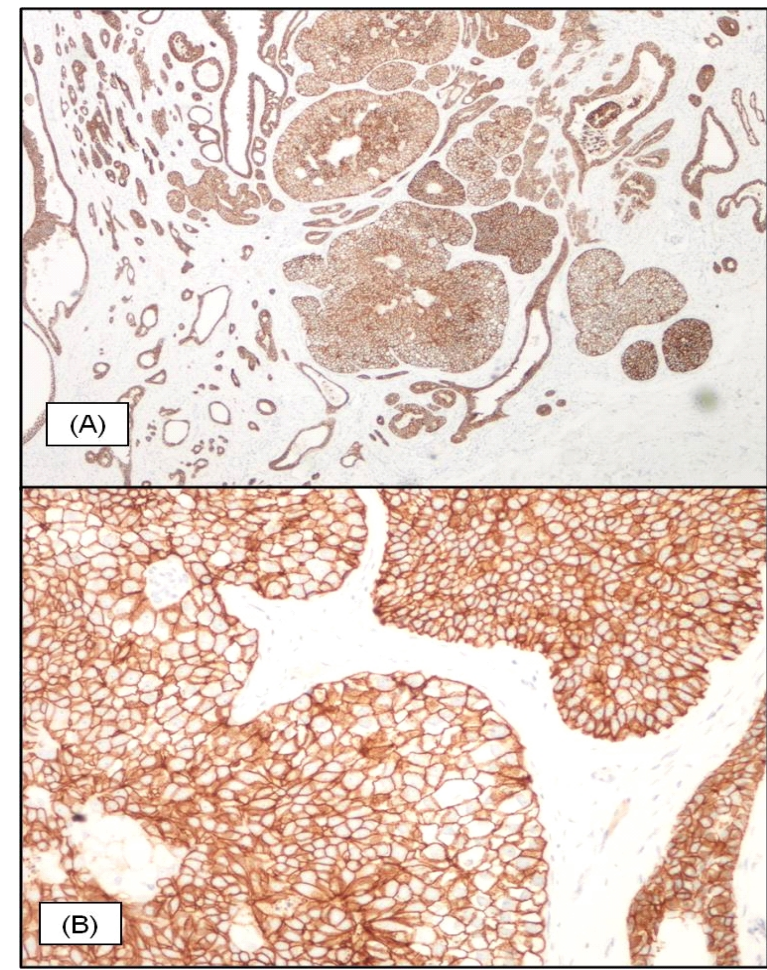

Figure 3: Immunohistochemistry of the breast lesion demonstrating heavy (+2) staining of the tissue for HER-2/Neu at both (a) 10x magnification and (b) 40x magnification.

The lesion was present as a single 3-4 mm sized focus within the complex fibroadenoma and $>2 \mathrm{~mm}$ from the nearest surgical margin. The DCIS was noted to have a solid type architectural pattern with apocrine and clear cell features and intermediate nuclear grade. No comedo histology was noted on any of the slides. Immunohistochemical studies performed on the tissue demonstrated over $95 \%$ of the cells to be estrogen receptor (ER) positive, while less than $5 \%$ progesterone receptor (PR) positive. In addition, the sample demonstrated heavy staining for HER-2/Neu receptor (Figure 3).

\section{Discussion}

The risk of cancer in women with Turner syndrome has been little studied. ${ }^{1}$ Yet it is an important area for consideration, because the hormonal abnormalities and treatments associated with this syndrome might affect the risk of hormone-related cancers. Moreover, the chromosomal abnormality itself might affect cancer risk. Knowledge of this risk is also important for patient and family education on the disease and its prognostic implications, and for clinical and screening practice during the follow-up of these patients. ${ }^{7}$

One might consider that the risk of breast cancer in TS patients would be relatively decreased, considering underdevelopment of breast tissue in most Turner patients and low endogenous estrogen concentrations. ${ }^{3}$ 
That assumption was factually confirmed in a recent study published by Schoemaker et al. ${ }^{7}$, who demonstrated that TS patients in their cohort had significantly decreased rates of breast neoplasms as compared with that of the general population. It is important to note, however, that the studied TS patients had only endogenous hormone levels, none having undergone HRT. The question of breast malignancies in the setting of long-term HRT remains therefore, uninvestigated in large-scale population studies.

This issue becomes relevant in the setting of the WHI trial results ${ }^{6}$, where hormone replacement therapy in general has been called into question. While the WHI study investigated healthy postmenopausal female as having an increased risk of breast cancer in women who received more than 5 years of HRT, its results were applied to all women regardless of age or hormonal status. Recent studies by Bösze et $a l^{8}$, as well as by Warren et $a l^{4}$, claimed this to be an inappropriate extrapolation. They point out that levels of estrogen and progesterone in TS patients undergoing HRT are much lower than in normal menstruating woman of similar age group. Especially since the use of oral contraceptives, which are much higher in estrogen content, have not shown the same risk. ${ }^{9}$ Similarly, they claim that the risks cannot be extended to adolescents and young adults with TS, given the difference in age between the populations and the relative risks versus benefits of hormone replacement therapy for each age group. Since women with TS, who are denied exposure to sex hormones have substantial risk or morbidity in the areas of growth, osteoporosis, and psychosocial development, these authors advocate for generalized introduction of HRT among individuals with TS., ${ }^{4,8}$

In light of those studies, we find it especially important to publish our case as it demonstrates a unique case of breast malignancy in a Turner patient on long-term HRT. Consequently, as it appears in a patient meant to be at very low risk for such a malignancy, it raises important concerns in relation to plausible causality between HRT and breast neoplasms. The astute clinician must, therefore, be aware and exercise appropriate level of clinical suspicion in this otherwise protected population.

\section{Conclusion}

As adolescents with TS approach menopausal age, and as more long-term data become available, estrogenprogesterone replacement risks and benefits may become clearer. As such, our case report might be a sentinel event demonstrating that long-term hormone replacement in these individuals carries a higher risk of breast malignancies and pre-malignant lesions than previously considered. We propose that further studies are needed to explore the possible risk of long-term hormone replacement therapy as an underlying cause of breast neoplasms in TS patients.

\section{Conflict of interest}

The authors declare that they have no conflicts of interest. The authors alone are responsible for the content and writing of the paper.

\section{References}

1. Stochholm K, Juul S, Juel K, et al. Prevalence, incidence, diagnostic delay, and mortality in Turner syndrome. J Clin Endocrinol Metab. 2006;91:3897-902.

2. Gravholt $\mathrm{CH}$. Epidemiological, endocrine and metabolic features in Turner syndrome. Eur J Endocrinol. 2004; 151:657-87.

3. Elsheikh M, Dunger DB, Conway GS, Wass JA. Turner's syndrome in adulthood. Endocr Rev. 2002;23:120-40.

4. Warren MP, Chua A. Appropriate use of estrogen replacement therapy in adolescents and young adults with Turner syndrome and hypopituitarism in light of the Women's Health Initiative. Growth Horm IGF Res. 2006;16 Suppl A:S98-102.

5. Giordano R, Forno D, Lanfranco F, et al. Metabolic and cardiovascular outcomes in a group of adult patients with Turner's syndrome under hormonal replacement therapy. Eur J Endocrinol. 2011; 164:819-26.

6. Rossouw JE, Anderson GL, Prentice RL, et al. Risk and benefits of estrogen plus progestin in healthy postmenopausal women: principal results from the Women's Health Initiative randomized controlled trial. JAMA. 2002; 288:321-33.

7. Schoemaker MJ, Swerdlow AJ, Higgins CD, et al. Cancer incidence in women with Turner syndrome in Great Britain: a national cohort study. Lancet Oncol. 2008; 9:239-46.

8. Bösze P, Tóth A, Török M. Hormone replacement and the risk of breast cancer in Turner's syndrome. N Engl J Med. 2006; 355:2599-600.

9. Collaborative Group on Hormonal Factors in Breast Cancer. Breast cancer and hormonal contraceptives: collaborative reanalysis of individual data on 53,297 women with breast cancer and 100,239 women without breast cancer from 54 epidemiological studies. Lancet. 1996; 347:1713-27. 